\title{
Single-Walled Carbon Nanotubes Template the One-Dimensional Ordering of a Polythiophene Derivative
}

Atsushi Ikeda, ${ }^{*}$ Kazuyuki Nobusawa, Tomoe Hamano, and Jun-ichi Kikuchi

Graduate School of Materials Science, Nara Institute of Science and Technology, 8916-5 Takayama, Ikoma 630-0192, Japan

\section{Supporting Information}
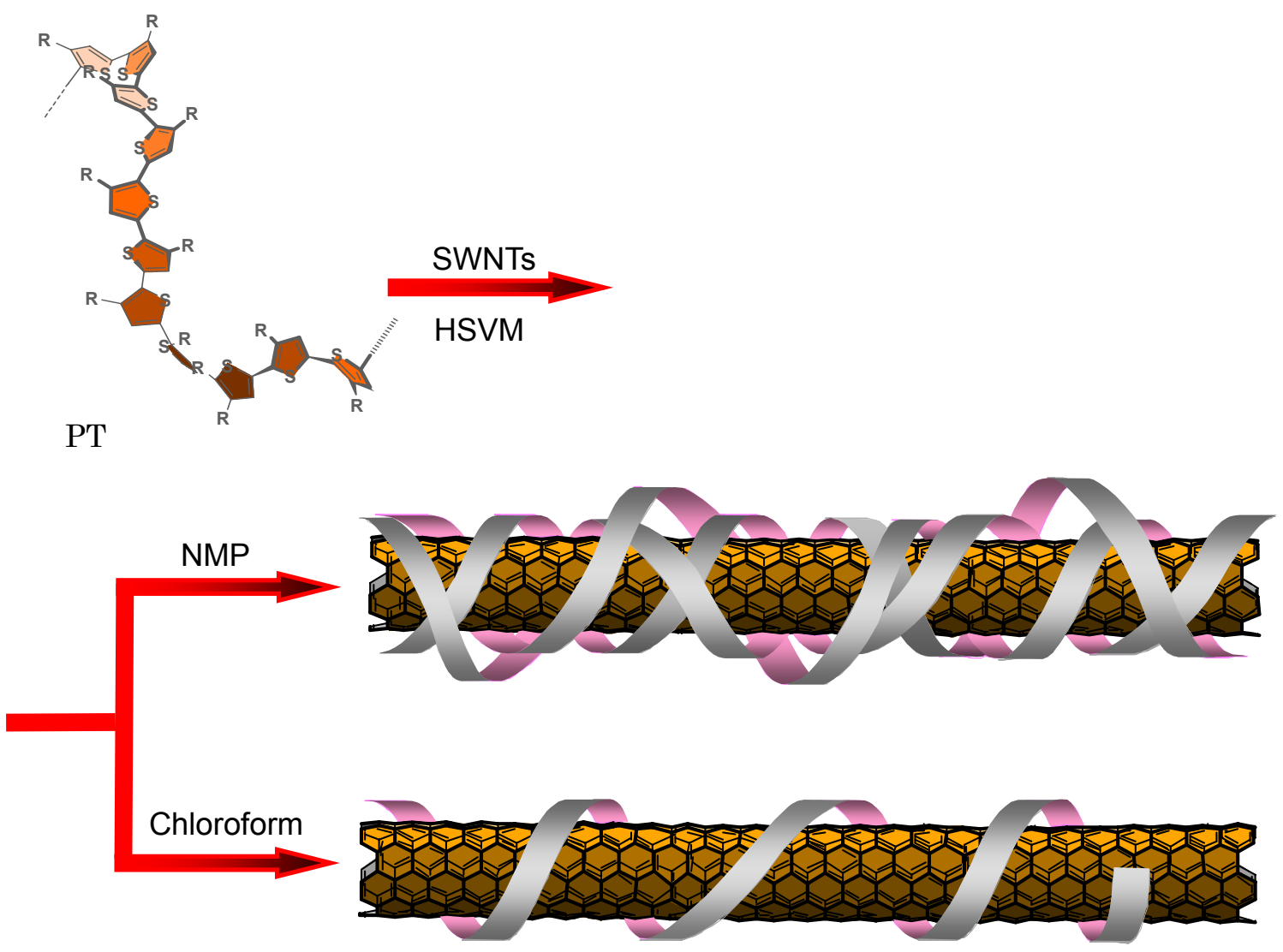

Scheme S1. Schematic illustration of an SWNT.PT complex because in Figure 2A, the SWNT surface was coated by several PT chains and in Figure 2B, part of the SWNT surface was cropped out. 


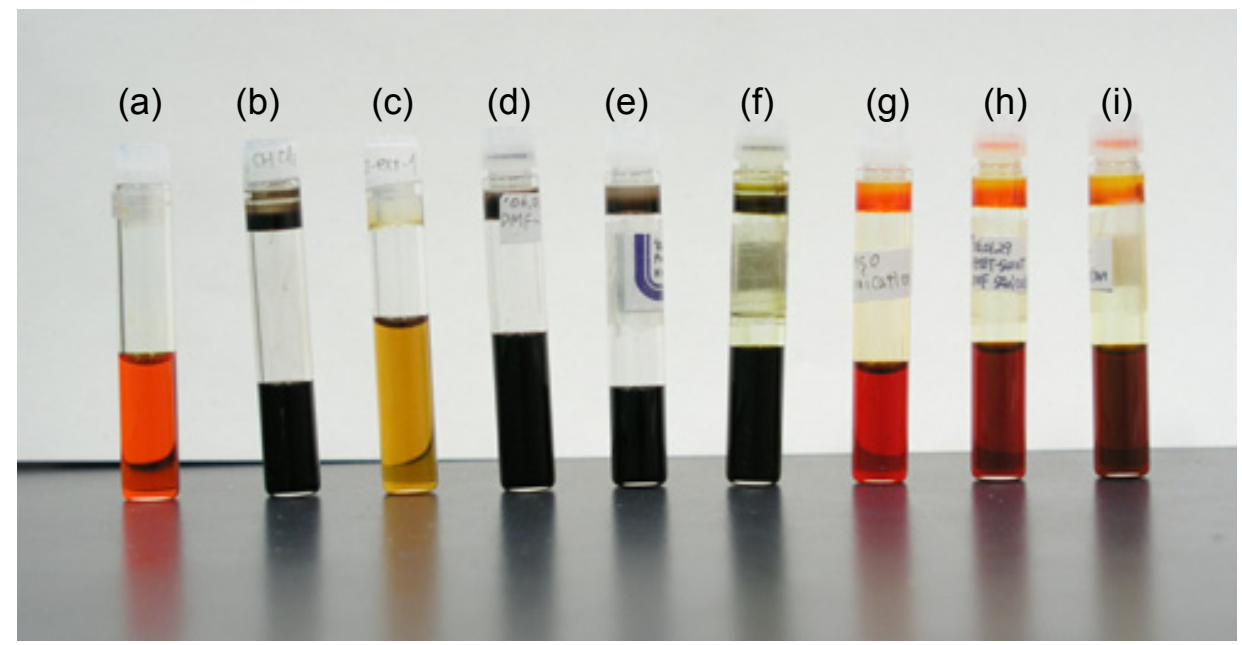

Figure S1. Photographs of vials showing PT in (a) NMP, the SWNT.PT complexes in (b) chloroform, (c) DMSO, (d) DMF, and (e) NMP, by HSVM, and the SWNT.PT complexes in (f) chloroform, (g) DMSO, (h) DMF, and (i) NMP, by the sonication method. 


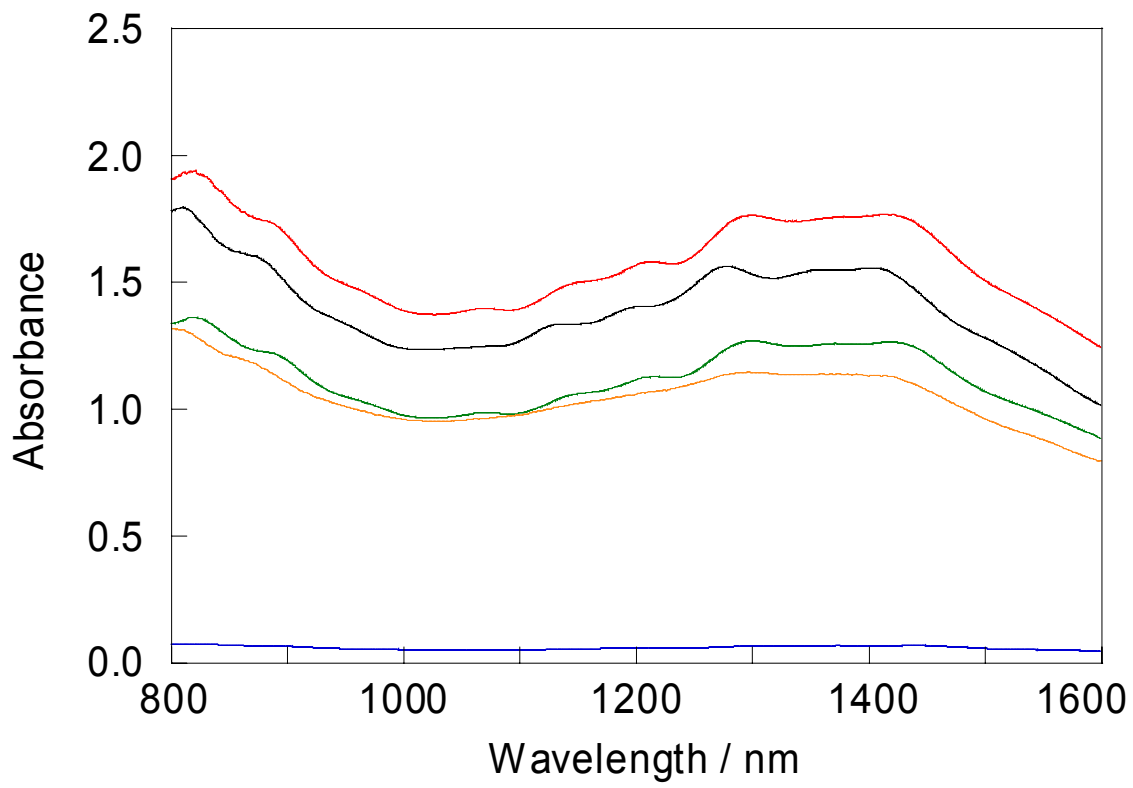

Figure S2. Near-IR spectra of the SWNT.PT complexes prepared by HSVM in (a) chloroform (black line), (b) NMP (red line), (c) DMF (green line), and (d) DMSO (blue line) and (e) the SWNT.PVP complex in NMP (orange line) at $25{ }^{\circ} \mathrm{C}$ (1-mm cell) [N.B.: the spectrum of the SWNT.PVP complex has been expanded $(\times 10)]$. 

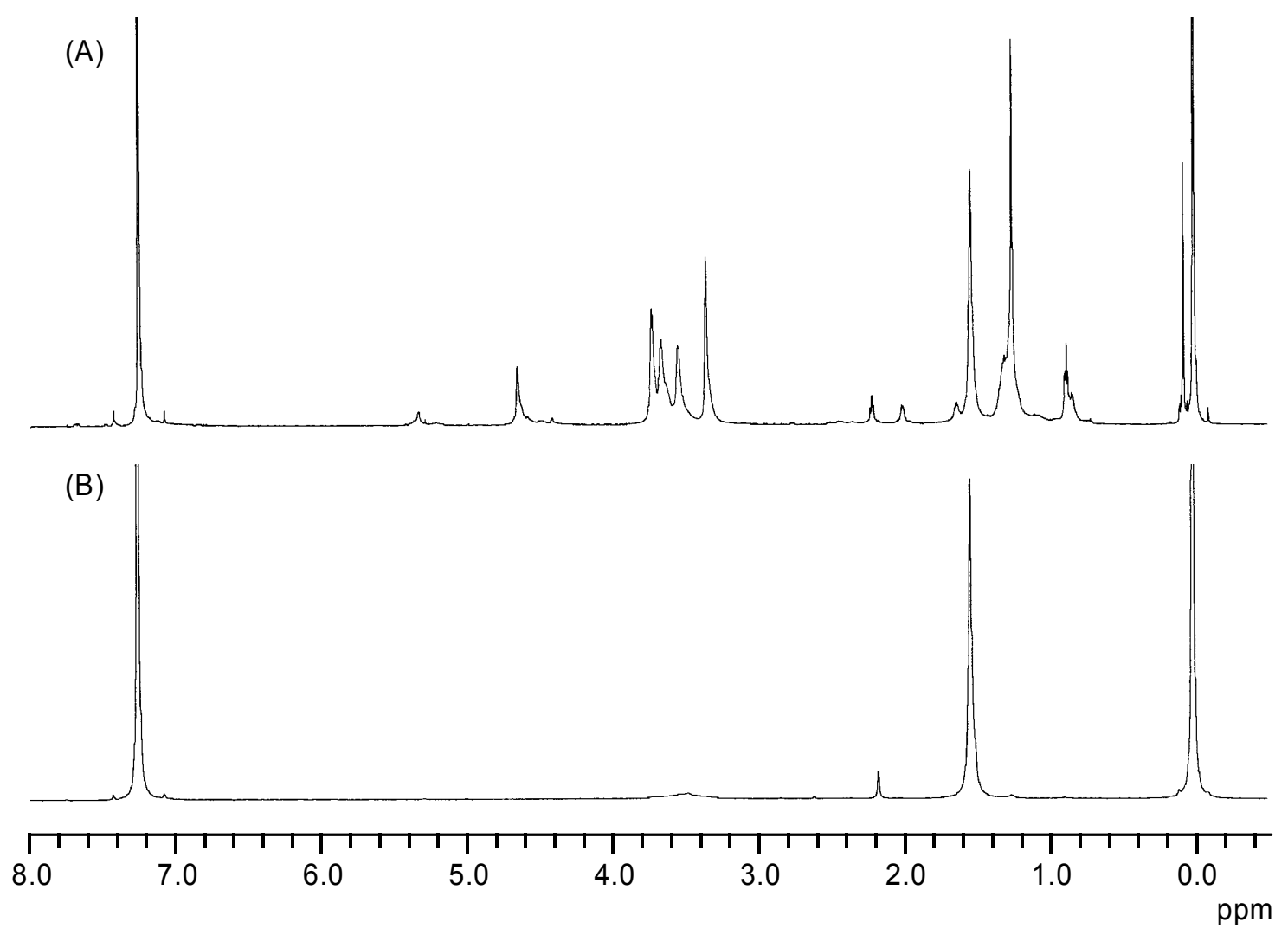

Figure S3. ${ }^{1} \mathrm{H}$ NMR spectra of (A) PT and (B) the SWNT.PT complex in $\mathrm{CDCl}_{3}$ at $25^{\circ} \mathrm{C}$. 